VOL. 68 (2003) [205-212]

\title{
CHARACTERISATIONS OF BLOCH FUNCTIONS IN THE UNIT BALL OF $\mathbb{C}^{n}$, I
}

\section{ZeNGJian LOU AND Hasi Wulan}

Weighted Hadamard product characterisations of Bloch functions in the unit ball of $\mathbb{C}^{n}$ are studied. In particular, we prove that $f$ belongs to the Bloch space $\mathcal{B}\left(B_{n}\right)$ if and only if the non-weighted Hadamard products of $f$ and $g$ belong to $B M O A\left(U^{n}\right)$ for all $f$ in $G_{0}^{1}\left(B_{n}\right)$, a subspace of the Hardy space $H^{1}\left(B_{n}\right)$.

\section{INTRODUCTION}

Hadamard products and related coefficient multiplier problems for spaces of holomorphic functions in the unit disk have been studied extensively in many papers; see, for example, [9]. In the higher dimensional extension of such a study, Burbea and $\mathrm{Li}$ [3] obtained recently some new descriptions of multi-index coefficient multiplier problems and the properties of weighted Hadamard products of holomorphic functions of several complex variables. In this note, for the higher dimensional setting of these questions, we prove new characterisations of spaces of multipliers of holomorphic functions in the unit ball, which characterise Bloch functions by weighted Hadamard products.

We first set up some basic notations which shall be used in the note, most of them can be found in $[3]$. Let $\mathbb{C}^{n}$ denote the $n$-dimensional vector space. For $z$ $=\left(z_{1}, \ldots, z_{n}\right) \in \mathbb{C}^{n}, \alpha=\left(\alpha_{1}, \ldots, \alpha_{n}\right) \in \mathbb{Z}_{+}^{n}$, set

$$
\begin{gathered}
\partial_{j}=\frac{\partial}{\partial z_{j}}, \quad 1 \leqslant j \leqslant n, \\
\partial^{\alpha}=\partial_{1}^{\alpha_{1}} \ldots \partial_{n}^{\alpha_{n}}, \quad \nabla=\left(\partial_{1}, \ldots, \partial_{n}\right),
\end{gathered}
$$

$z \cdot \xi=\left(z_{1} \xi_{1}, \ldots, z_{n} \xi_{n}\right)$ and $\langle z, \xi\rangle=z_{1} \bar{\xi}_{1}+\cdots+z_{n} \bar{\xi}_{n}$ if $\xi=\left(\xi_{1}, \ldots, \xi_{n}\right) \in \mathbb{C}^{n}$.

Let $B_{n}$ and $U^{n}$ denote respectively the unit ball and the unit polydisk in $\mathbb{C}^{n}$. For a complete Rheinhardt domain $\Omega$ of $\mathbb{C}^{n}$, by $H(\Omega)$ we denote the space of all holomorphic functions in $\Omega$. Then any $f \in H(\Omega)$ has a unique power series

$$
f(z)=\sum_{\alpha} a_{\alpha} z^{\alpha}, \quad z \in \Omega
$$

Received 3rd December, 2002

The first author was supported by Australian Government through the Australian Research Council and would like to express his thanks to the Australian Government and the Australian National University for financial support. The second author is supported in part by a grant from the NSFGP (No. 010446).

Copyright Clearance Centre, Inc. Serial-fee code: 0004-9727/03 \$A2.00+0.00. 
with $a_{\alpha}=\frac{\partial^{\alpha} f(0)}{\alpha !}, z^{\alpha}=z_{1}^{\alpha_{1}} \ldots z_{n}^{\alpha_{n}}, \alpha \in \mathbb{Z}_{+}^{n}$. So $H(\Omega)$ may be regarded as a space of multi-index sequences $\left\{a_{\alpha}\right\}$.

For $f(z)=\sum_{\alpha} a_{\alpha} z^{\alpha}, g(z)=\sum_{\alpha} b_{\alpha} z^{\alpha} \in H\left(B_{n}\right)$ and $q \geqslant 0$, we define the $q$ Hadamard product of $f$ and $g$ by

$$
(f * g)_{q}(z)=\sum_{\alpha} \omega_{\alpha}(q) a_{\alpha} b_{\alpha} z^{\alpha}
$$

where

$$
\omega_{\alpha}(q)=\frac{\alpha ! \Gamma(n+q)}{\Gamma(n+q+|\alpha|)}, \quad \alpha \in \mathbb{Z}_{+}^{n} .
$$

The interesting feature of this product is that it is lying not only in $H\left(B_{n}\right)$ but also in $H\left(U^{n}\right)$ and

$$
(f * g)_{q}(z)=\left\langle f, g_{\bar{z}}^{*}\right\rangle_{q}:=\int f(r \xi) g(z \cdot \bar{\xi}) d v_{q}(\xi)
$$

for any $z \in U^{n}$, where

$$
\begin{aligned}
f_{\xi}(z) & =f(\xi \cdot z) \\
g^{*}(z) & =\overline{g(\bar{z})} \\
d v_{q}(z) & =\frac{1}{\pi^{n}} \frac{\Gamma(n+q)}{\Gamma(q)}\left(1-|z|^{2}\right)^{q-1} d v(z)
\end{aligned}
$$

and $d v$ denotes the usual volume of Lebesgue measure on $\mathbb{C}^{n}$ (see [3, Proposition 3.2]).

Let $X$ and $Y$ be two vector spaces of multi-index sequences. A multi-index sequence $\left\{\lambda_{\alpha}\right\}$ is said to be a multiplier from $X$ to $Y$, if $\left\{\lambda_{\alpha} x_{\alpha}\right\} \in Y$ whenever $\left\{x_{\alpha}\right\} \in X$. The set of all multipliers from $X$ to $Y$ is denoted by $(X, Y)$. A multi-index sequence $\left\{\lambda_{\alpha}\right\}$ is said to be a $q$-multiplier, $q \geqslant 0$, from $X$ to $Y$ if $\left\{\lambda_{\alpha} \omega_{\alpha}(q) x_{\alpha}\right\} \in Y$ whenever $\left\{x_{\alpha}\right\} \in X$. The set of all $q$-multipliers from $X$ to $Y$ is denoted by $(X, Y)_{q}$. Recently Burbea and Li have shown in [3] that

$$
\mathcal{B}\left(B_{n}\right)=\left(A_{q}^{1}\left(B_{n}\right), B M O A\left(U^{n}\right)\right)_{q}
$$

where $\mathcal{B}\left(B_{n}\right)$ is the Bloch space and $B M O A\left(U^{n}\right)$ the space of functions in $H\left(U^{n}\right)$ with bounded mean oscillation on the torus $T^{n}:=\partial U^{n}$ and $A_{q}^{1}\left(B_{n}\right)$ is the Bergman-type space $\left(A_{0}^{1}\left(B_{n}\right)=H^{1}\left(B_{n}\right)\right.$, the Hardy space). In this note we prove that the space $A_{q}^{1}\left(B_{n}\right)$ in (3) can be replaced by its restricted subspaces (Theorem 1).

We next introduce some further notations and recall some function theoretic concepts. For $s>0$, let

$$
\left(\mathcal{D}^{s} f\right)(z)=\sum_{\alpha}(|\alpha|+1)^{s} a_{\alpha} z^{\alpha}
$$


be the fractional derivative of $f(z)=\sum_{\alpha} a_{\alpha} z^{\alpha}$ of order $s$.

The Bloch space on the unit ball, denoted by $\mathcal{B}\left(B_{n}\right)$, is defined as the space of $f \in H\left(B_{n}\right)$ for which

$$
\|f\|_{\mathcal{B}\left(B_{n}\right)}=|f(0)|+\sup _{z \in B_{n}}\left(1-|z|^{2}\right)|\nabla f(z)|<\infty .
$$

The small Bloch space $\mathcal{B}_{0}\left(B_{n}\right)$ is the $\mathcal{B}\left(B_{n}\right)$-closure of the holomorphic polynomials in $z \in \mathbb{C}^{n}$ (see [11] for more information). It is easy to see that $f \in \mathcal{B}\left(B_{n}\right)$ if and only if

$$
\sup _{z \in B_{n}}\left(1-|z|^{2}\right)^{s}\left|\left(\mathcal{D}^{s} f\right)(z)\right|<\infty, \quad s>0
$$

(refer [7, Theorem 2.1]). Similarly, the Bloch space on the unit polydisk $U^{n}$, denoted by $\mathcal{B}\left(U^{n}\right)$, is the space of $f \in H\left(U^{n}\right)$ satisfying

$$
\|f\|_{\mathcal{B}\left(U^{n}\right)}=\sup _{z \in U^{n}} \sum_{j=1}^{n}\left(1-\left|z_{j}\right|^{2}\right)\left|f(z)+n z_{j} \partial_{j} f(z)\right|<\infty .
$$

The small Bloch space $\mathcal{B}_{0}\left(U^{n}\right)$ is the $\mathcal{B}\left(U^{n}\right)$-closure of the holomorphic polynomials in $z \in \mathbb{C}^{n}$.

In order to give the definition of $B M O A$ space on the unit polydisk $U^{n}$, we first define differential operator $D^{n}$ of order $n$ with $D^{1}=d / d z$. Let $\delta: \mathbb{Z} \rightarrow \mathbb{Z}_{+}$ be defined by $\delta(m)=\max (m, 0), m \in \mathbb{Z}$. For $\alpha=\left(\alpha_{1}, \ldots, \alpha_{n}\right) \in \mathbb{Z}_{+}^{n}$, let $\delta(\alpha)$ $=\left(\delta\left(\alpha_{1}\right), \ldots, \delta\left(\alpha_{n}\right)\right) \in \mathbb{Z}_{+}^{n}$ and $\pi(\alpha)=\prod_{i=1}^{n}\left\{\alpha_{i}: \alpha_{i} \neq 0\right\}$. We define

$$
\left(D^{n} f\right)(z)=\sum_{\alpha} \pi(\alpha) a_{\alpha} z^{\delta(\alpha-1)} .
$$

$B M O A\left(U^{n}\right)$ is the space of functions $f$ in $H\left(U^{n}\right)$ with bounded mean oscillation on the torus $T^{n}$ :

$$
\|f\|_{B M O A\left(U^{n}\right)}^{2}=|f(0)|^{2}+\sup _{\xi \in U^{n}} \int_{U^{n}}\left|\left(D^{n} f\right)(z)\right|^{2} \prod_{j=1}^{n} \frac{\left(1-\left|z_{j}\right|^{2}\right)\left(1-\left|\xi_{j}\right|^{2}\right)}{\left|1-z_{j} \bar{\xi}_{j}\right|^{2}} \frac{d v(z)}{\pi^{n}}<\infty
$$

(see $[4$, p. 238] for $n=1)$. Equipped with the norm $\|\cdot\|_{B M O A\left(U^{n}\right)}, B M O A\left(U^{n}\right)$ is a Banach space. The closure in $B M O A\left(U^{n}\right)$ of the holomorphic polynomials in $z \in \mathbb{C}^{n}$ gives rise to the space of functions in $H\left(U^{n}\right)$ with vanishing mean oscillation on $T^{n}$ and is denoted by $V M O A\left(U^{n}\right)$.

We now introduce a new quasi-Banach space,

$$
G_{q}^{1}\left(B_{n}\right)=\left\{f \in H\left(B_{n}\right):\|f\|_{G_{q}^{1}\left(B_{n}\right)}<\infty\right\}, \quad 0 \leqslant q<\infty,
$$

where

$$
\|f\|_{G_{q}^{1}\left(B_{n}\right)}=\int_{B_{n}}\left|\mathcal{D}^{1} f(z)\right|\left(1-|z|^{2}\right) d v_{q}(z)<\infty
$$




\section{THEOREM AND ITS PROOF}

Now we state our main theorem which gives a new characterisation of the Bloch space $\mathcal{B}\left(B_{n}\right)$ with $q$-Hadamard products.

THEOREM 1. For any $q \geqslant 0$, we have

$$
\mathcal{B}\left(B_{n}\right)=\left(G_{q}^{1}\left(B_{n}\right), B M O A\left(U^{n}\right)\right)_{q} .
$$

Proof: Let $A\left(U^{n}\right)$ denote the space of functions which holomorphic in $U^{n}$ and continuous on $\overline{U^{n}}$. It is well-known that $A\left(U^{n}\right) \subset B M O A\left(U^{n}\right)$. To prove

$$
\mathcal{B}\left(B_{n}\right) \subset\left(G_{q}^{1}\left(B_{n}\right), B M O A\left(U^{n}\right)\right)_{q}
$$

it suffice to show that

$$
\mathcal{B}\left(B_{n}\right) \subset\left(G_{q}^{1}\left(B_{n}\right), A\left(U^{n}\right)\right)_{q} .
$$

For $q>0$. Let $A_{q}^{1}\left(B_{n}\right)$ be the space of $f \in H\left(B_{n}\right)$ for which

$$
\|f\|_{A_{q}^{1}\left(B_{n}\right)}=\int_{B_{n}}|f(z)| d v_{q}(z)<\infty
$$

([3]). By $\left[\mathbf{1 0}\right.$, Theorem 1(b)], $G_{q}^{1}\left(B_{n}\right) \subset A_{q}^{1}\left(B_{n}\right)$. Then it is sufficient to prove that

$$
\mathcal{B}\left(B_{n}\right) \subset\left(A_{q}^{1}\left(B_{n}\right), A\left(U^{n}\right)\right)_{q}
$$

where we used [1, Lemma 1].

Suppose that $\Gamma_{q}\left(B_{n}\right)$ is the dual of $A_{q}^{1}\left(B_{n}\right)$ with respect to the $q$-pairing. Then $\Gamma_{q}\left(B_{n}\right)=\mathcal{B}\left(B_{n}\right)[2]$. Applying [3, Propositions 3.1 and 3.3], (2) gives

$$
\left|(f * g)_{q}(z)\right| \leqslant\|g\|_{\Gamma_{q}\left(B_{n}\right)}\left\|f_{\bar{z}}^{*}\right\|_{A_{q}^{1}\left(B_{n}\right)} \leqslant C\|g\|_{B\left(B_{n}\right)}\|f\|_{A_{q}^{1}\left(B_{n}\right)} .
$$

For $f \in A_{q}^{1}\left(B_{n}\right), z \in \overline{U^{n}}$, the correspondence $z \rightarrow f_{z} \in A_{q}^{1}\left(B_{n}\right)$ is continuous. That is $\left\|f_{z_{1}}-f_{z_{2}}\right\|_{A_{q}^{1}\left(B_{n}\right)} \rightarrow 0$ as $\left|z_{1}-z_{2}\right| \rightarrow 0$ (refer [8, Lemma 4 (iii)]). Combining this with (4) yields

$$
\begin{aligned}
\left|(f * g)_{q}\left(z_{1}\right)-(f * g)_{q}\left(z_{2}\right)\right| & =\left|\left(\left(f_{z_{1}}-f_{z_{2}}\right) * g\right)_{q}(1)\right| \\
& \leqslant\left\|f_{z_{1}}-f_{z_{2}}\right\|_{A_{q}^{1}\left(B_{n}\right)}\|g\|_{B\left(B_{n}\right)} \\
& \rightarrow 0
\end{aligned}
$$

as $\left|z_{1}-z_{2}\right| \rightarrow 0$. Hence $(f * g)_{q} \in A\left(U^{n}\right)$ for all $f \in A_{q}^{1}\left(B_{n}\right)$. 
For $q=0$. Let $f(z)=\sum_{\alpha} a_{\alpha} z^{\alpha} \in G_{0}^{1}\left(B_{n}\right), g(z)=\sum_{\alpha} b_{\alpha} z^{\alpha} \in \mathcal{B}\left(B_{n}\right)$, we have

$$
\sum_{\alpha}(|\alpha|+1)^{2} r^{2|\alpha|} a_{\alpha} b_{\alpha} \omega_{\alpha}(0) z^{\alpha}=\int_{\partial B_{n}} \mathcal{D}^{1} f(r \xi) \mathcal{D}^{1} g(r z \cdot \bar{\xi}) d v(\xi) .
$$

Noting that $\int_{0}^{1} r^{2 n+1} \log 1 / r d r=1 /\left(4(n+1)^{2}\right)$. We obtain

$$
\begin{aligned}
\left|(f * g)_{0}(z)\right| & =\left|4 \int_{0}^{1} r \log \frac{1}{r} \int_{\partial B_{n}} \mathcal{D}^{1} f(r \xi) \mathcal{D}^{1} g(r z \cdot \bar{\xi}) d v(\xi) d r\right| \\
& \leqslant 4 \int_{0}^{1} \int_{\partial B_{n}}(1-r)\left|\mathcal{D}^{1} f(r \xi)\right| \mathcal{D}^{1} g(r z \cdot \bar{\xi}) \mid d v(\xi) d r \\
& \leqslant 4\|g\|_{\mathcal{B}\left(B_{n}\right)}\|f\|_{G_{0}^{1}\left(B_{n}\right)} .
\end{aligned}
$$

It is easy to see that

$$
\left\|(f * g)_{0}\left(z_{1}\right)-(f * g)_{0}\left(z_{2}\right)\right\|_{G_{0}^{1}\left(B_{n}\right)} \rightarrow 0
$$

as $\left|z_{1}-z_{2}\right| \rightarrow 0$. Thus $(f * g)_{0} \in A\left(U^{n}\right)$ for all $f \in G_{0}^{1}\left(B_{n}\right)$.

We now prove $\left(G_{q}^{1}\left(B_{n}\right), B M O A\left(U^{n}\right)\right)_{q} \subset \mathcal{B}\left(B_{n}\right)$ by showing that $\left(G_{q}^{1}\left(B_{n}\right)\right.$, $\left.\mathcal{B}\left(U^{n}\right)\right)_{q} \subset \mathcal{B}\left(B_{n}\right)$. For $g(z)=\sum_{\alpha} b_{\alpha} z^{\alpha}$, we defined a linear operator

$$
T_{g}: G_{q}^{1}\left(B_{n}\right) \rightarrow \mathcal{B}\left(U^{n}\right)
$$

by $T_{g}(f)=(f * g)_{q}$. So $T_{g}$ is a bounded linear operator from $G_{q}^{1}\left(B_{n}\right)$ to $\mathcal{B}\left(U^{n}\right)$ and

$$
\left\|(f * g)_{q}\right\|_{B\left(U^{n}\right)} \leqslant C_{g}\|f\|_{G_{q}^{1}\left(B_{n}\right)}
$$

for all $f \in G_{q}^{1}\left(B_{n}\right)$, where $C_{g}$ is a constant depending on $g$ ([3, Lemma 3.4]).

Let $a \in \mathbb{C}$ and $m \in Z_{+},(a)_{m}$ stands for 1 if $m=0$ and $a(a+1) \ldots(a+m-1)$ if $m>0$, let $\lambda \in U$ and

$$
G_{a, b}(\lambda):=\sum_{m=0}^{\infty}(m+1)^{-b} \frac{(a)_{m}}{m !} \lambda^{m}, \quad a, b \in \mathbb{C} .
$$

Setting $f:=G_{n+q,-b+1}(\langle\cdot r \bar{\xi}\rangle), \xi \in \partial B_{n}, 0 \leqslant r<1,1<b$, then $\mathcal{D}^{1} f$ $=G_{n+q,-b}(\langle\cdot, r \bar{\xi}\rangle)$. For $\eta \in U^{n}$, we have

$$
\begin{aligned}
h(\eta) & :=(f * g)_{q}(\eta) \\
& =\int g(\eta \cdot \bar{z}) G_{n+q,-b+1}(\langle z, r \bar{\xi}\rangle) d v_{q}(z) \\
& =\sum_{\alpha}(|\alpha|+1)^{b-1} b_{\alpha}(r \xi \eta)^{\alpha} \\
& =\mathcal{D}^{b-1} g_{r \xi}(\eta) .
\end{aligned}
$$


This implies

$$
\mathcal{D}^{1} h(r 1)=\mathcal{D}^{b} g\left(r^{2} \xi\right)
$$

Using [3, Proposition 3.5(iii)] we have $G_{n+q,-b}(\lambda)=(1-\lambda)^{-(n+q+b)} F(\lambda), \lambda \in U$, where $F$ satisfies

$$
\sup _{z \in B_{1}}\left(1-|z|^{2}\right)^{m-n-q-b}\left|\mathcal{D}^{n+q+b} F(z)\right|<\infty
$$

for some $m>0$ (see [3, p. 246]). Proposition 3.5(ii) gives

$$
\begin{aligned}
\|f\|_{G_{q}^{1}\left(B_{n}\right)} & \leqslant\|F\|_{\infty} \int|1-\langle z, r \bar{\xi}\rangle|^{-(n+q+b)}\left(1-|z|^{2}\right) d v_{q}(z) \\
& \leqslant C\|F\|_{\infty}\left(1-r^{2}\right)^{1-b}
\end{aligned}
$$

where $C$ is a constant depending only on $q, n$ and $b$. Combining (6) with (7) and (8) we get

$$
\left|\mathcal{D}^{b} g\left(r^{2} \xi\right)\right| \leqslant C C_{g}\|F\|_{\infty}\left(1-r^{2}\right)^{-b}, \quad \xi \in \partial B_{n}, 0<r<1 .
$$

It follows that $g \in \mathcal{B}\left(B_{n}\right)$. The proof of Theorem 1 is finished. REMARKS.

(1) Let $q=0$ in Theorem 1 , we have

$$
\mathcal{B}\left(B_{n}\right)=\left(G_{0}^{1}\left(B_{n}\right), B M O A\left(U^{n}\right)\right)_{0}
$$

That is $f$ belongs to the Bloch space $\mathcal{B}\left(B_{n}\right)$ if and only if the nonweighted Hadamard products of $f$ and $g$ belong to $B M O A\left(U^{n}\right)$ for all $f$ in $G_{0}^{1}\left(B_{n}\right)$, a subspace of the Hardy space $H^{1}\left(B_{n}\right)$.

(2) When $n=1$ and $q=0$, Theorem 1 was proved in [5].

We next introduce the generalised mean Lipschitz space $\mathfrak{L}\left(U^{n}\right)$. For $z$ $=\left(z_{1}, \ldots, z_{n}\right)$, let $z_{(j)}=\left(z_{1}, \ldots, z_{j-1}\right) \in \mathbb{C}^{j-1}$ if $j>1$ and $z^{(j)}=\left(z_{j+1}, \ldots, z_{n}\right)$ $\in \mathbb{C}^{n-j}$ for $j<n$. So $\left(z_{(j)}, z^{(j)}\right) \in \mathbb{C}^{n-1}$ if $n>1$. If $f$ is a function defined in a $\mathbb{C}^{n}$-neighbourhood $N^{n}(z)$ of $z$, then $f\left(z_{(j)}, \cdot, z^{(j)}\right)$ is a function defined in $N_{j}^{n}(z)$ $=\left\{\ell \in \mathbb{C}:\left(z_{(j)}, \ell, z^{(j)}\right) \in N^{n}(z)\right\}$. Define $\mathfrak{L}\left(U^{n}\right)$ the space of $f \in H\left(U^{n}\right)$ with $\|f\|_{\mathcal{L}\left(U^{n}\right)}^{2}=|f(0)|^{2}+\sum_{j=1}^{n} \sup _{\left(z_{(j)}, z^{(j)}\right) \in U^{n-1}} \int_{0}^{1} M_{\infty}^{2}\left(r,\left(z_{(j)}, z^{(j)}\right): \partial_{j} f\right)\left(1-r^{2}\right) 2 r d r<\infty$ where $M_{\infty}\left(r,\left(z_{(j)}, z^{(j)}\right): f\right)=\sup \left\{\left|f\left(z_{(j)}, r \ell, z^{(j)}\right)\right|: \ell \in \partial B_{1}\right\}$. Equipped with the norm $\|\cdot\|_{\mathfrak{L}\left(U^{n}\right)}, \mathfrak{L}\left(U^{n}\right)$ is a Banach space. Let $H^{\infty}\left(U^{n}\right)$ denote the space of all bounded holomorphic functions in $U^{n}$. The proof of Theorem 1 implies the following. 
Corollary 2. For $q \geqslant 0$

$$
\begin{aligned}
\mathcal{B}\left(B_{n}\right) & =\left(G_{q}^{1}\left(B_{n}\right), A\left(U^{n}\right)\right)_{q}=\left(G_{q}^{1}\left(B_{n}\right), H^{\infty}\left(U^{n}\right)\right)_{q} \\
& =\left(G_{q}^{1}\left(B_{n}\right), \mathfrak{L}\left(U^{n}\right)\right)_{q}=\left(G_{q}^{1}\left(B_{n}\right), V M O A\left(U^{n}\right)\right)_{q} \\
& =\left(G_{q}^{1}\left(B_{n}\right), \mathcal{B}_{0}\left(U^{n}\right)\right)_{q}=\left(G_{q}^{1}\left(B_{n}\right), \mathcal{B}\left(U^{n}\right)\right)_{q} .
\end{aligned}
$$

Proof: In fact, the proof of Theorem 1 implies that

$$
\mathcal{B}\left(B_{n}\right)=\left(G_{q}^{1}\left(B_{n}\right), A\left(U^{n}\right)\right)_{q}=\left(G_{q}^{1}\left(B_{n}\right), \mathcal{B}\left(U^{n}\right)\right)_{q} .
$$

Noting that $A\left(U^{n}\right) \subset H^{\infty}\left(U^{n}\right) \subset \mathcal{B}\left(U^{n}\right)$. We have

$$
\mathcal{B}\left(B_{n}\right)=\left(G_{q}^{1}\left(B_{n}\right), H^{\infty}\left(U^{n}\right)\right)_{q} .
$$

From [3, Theorem 5.6], $\mathcal{B}\left(B_{n}\right) \subset\left(A_{q}^{1}\left(B_{n}\right), \mathfrak{L}\left(U^{n}\right)\right)_{q}$. Combining this with the inclusion $G_{q}^{1}\left(B_{n}\right) \subset A_{q}^{1}\left(B_{n}\right)$, we obtain

$$
\mathcal{B}\left(B_{n}\right) \subset\left(G_{q}^{1}\left(B_{n}\right), \mathfrak{L}\left(U^{n}\right)\right)_{q}
$$

Since $\mathfrak{L}\left(U^{n}\right) \subset \operatorname{VMOA}\left(U^{n}\right)\left(\left[3\right.\right.$, Proposition 2.4]) and $\operatorname{VMOA}\left(U^{n}\right) \subset \mathcal{B}_{0}\left(U^{n}\right)$ $\subset \mathcal{B}\left(U^{n}\right)$, we have

$$
\mathcal{B}\left(B_{n}\right)=\left(G_{q}^{1}\left(B_{n}\right), \mathfrak{L}\left(U^{n}\right)\right)_{q}=\left(G_{q}^{1}\left(B_{n}\right), V M O A\left(U^{n}\right)\right)_{q}=\left(G_{q}^{1}\left(B_{n}\right), \mathcal{B}_{0}\left(U^{n}\right)\right)_{q} .
$$

Corollary 2 is proved.

In particular, Corollary 2 gives

$$
\mathcal{B}\left(B_{n}\right)=\left(G_{0}^{1}\left(B_{n}\right), A\left(U^{n}\right)\right)_{0}=\left(G_{0}^{1}\left(B_{n}\right), \mathcal{B}\left(U^{n}\right)\right)_{0}
$$

which is a result in [6]. When $G_{q}^{1}\left(B_{n}\right)$ is replaced by $A_{q}^{1}\left(B_{n}\right)$, Corollary 2 was proved by Burbea and $\mathrm{Li}$ in [3].

\section{REFERENCES}

[1] J.M. Anderson and A.L. Shields, 'Coefficient multipliers of Bloch functions', Trans. Amer. Math. Soc. 224 (1976), 255-265.

[2] F. Beatrous and J. Burbea, 'Holomorphic Sobolev spaces on the unit ball', Dissertationes Math. 276 (1989), 1-57.

[3] J. Burbea and S. Li, 'Weighted Hadamard products of holomorphic functions in the unit ball', Pacific J. Math. 168 (1995), 235-270.

[4] J.B. Garnett, Bounded analytic functions (Academic Press, New York, 1981). 
[5] Z. Lou, 'Multipliers of $H^{p}, G^{p}$ and Bloch spaces', Math. Japon. 36 (1991), 21-26.

[6] Z. Lou, 'Characterizations of Bloch functions on the unit ball of $\mathbb{C}^{n}$, Kodai Math. J. 16 (1993), 74-78.

[7] Z. Lou, 'Carleson measure characterizations of Bloch functions', Acta Math. Sinica 12 (1996), 175-184.

[8] Z. Lou, 'Coefficient multipliers of Bergman spaces $A^{p}$, II', Canad. Math. Bull. 40 (1997), 475-487.

[9] M. Mateljevic and M. Pavlovic, 'Multipliers of $H^{p}$ and BMOA', Pacific J. Math. 146 (1990), 71-84.

[10] J. Shi, 'On the rate of growth of the means $M_{g}$ of holomorphic and pluriharmonic functions on bounded symmetric domains of $\mathbb{C}^{n}$, J. Math. Anal. Appl. 126 (1987), 161-175.

[11] R. M. Timoney, 'Bloch functions in several complex variables, I', Bull. London Math. Soc. 12 (1980), 241-267.

\author{
Department of Mathematics \\ Shantou University \\ Shantou Guangdong 515063 \\ Peoples Republic of China \\ e-mail: zjlou@stu.edu.cn \\ and \\ Mathematical Sciences Institute \\ The Australian National University \\ Canberra ACT 0200 \\ Australia
}

\author{
Department of Mathematics \\ Shantou University \\ Shantou Guangdong 515063 \\ Peoples Republic of China \\ e-mail: wulan@stu.edu.cn
}

